\title{
Clinical phenotype of neonatal lupus erythematosus relates to autoantibody level and gender
}

\author{
S Venkatesan ${ }^{1 *}$, NG Lawrence ${ }^{2}$, C Carbone $^{2}$, E Jaeggi $^{3}$, ED Silverman² ${ }^{2}$ S Kamphuis ${ }^{1,2}$ \\ From 18th Pediatric Rheumatology European Society (PReS) Congress \\ Bruges, Belgium. 14-18 September 2011
}

\section{Background}

Neonatal Lupus Erythematodus (NLE) is a rare disease occurring in offspring from mothers with anti-Ro with or without anti-La antibodies. Much is known about the individual manifestations (congenital heart block (CHB), cutaneous rash, hematologic and hepatic laboratory abnormalities and macrocephaly) but it is unclear how these and other autoantibodies (anti-dsDNA, anti-RNP and anti-Sm antibodies) influence disease phenotype.

\section{Aim}

To analyze the frequency and characteristics of clinical phenotypes seen in NLE and relate these phenotypes to the quality and quantity of autoantibodies.

\section{Methods}

A cohort of 261 infants whose mothers had anti-Ro antibodies was followed prospectively at The Hospital for Sick Children. All infants underwent the first full clinical evaluation and laboratory testing between 4-12 weeks after birth.

\section{Results}

A small majority of infants $(150 / 261,57 \%)$ had one or more NLE manifestations consisting of 56 (37\%) with a cutaneous rash, 44 (29\%) had liver function abnormalities, 42 (28\%) had CHB, 41 (27\%) had neutropenia $\left(<1.0 \times 10^{9} / 1\right), 21(14 \%)$ had macrocephaly and $9(6 \%)$ had thrombocytopenia. CHB was an isolated finding in more than half of the cases (26/42), as was neutropenia (23/ 41); the other NLE manifestations most often presented in combination. Where the cohort of NLE patients had slightly more females (59\% versus $41 \%$ males), a larger majority of patients with $\mathrm{CHB}$ were females (74\%), but macrocephaly was seen more in males (62\%). The chance of developing NLE was associated with increasing levels of anti-Ro antibodies. Macrocephaly was only seen in infants that still had a positive anti-Ro titer when sampled after birth. Increasing levels of anti-La antibodies were associated primarily with cutaneous rash. Anti-dsDNA, anti-RNP and anti-Sm antibodies were present in less than $5 \%$ of infants and could not be related to clinical phenotype.

\section{Conclusion}

In this large cohort of prospectively followed infants from mothers with anti-Ro antibodies, a majority developed NLE. The clinical phenotype of NLE appeared to be related to gender and to the quantity of anti-Ro and -La antibodies.

\section{Author details \\ 'Department of Rheumatology, Sophia's Children Hospital, Erasmus University MC Rotterdam, The Netherlands. ${ }^{2}$ Department of Rheumatology, The Hospital for Sick Children, Toronto, Canada. ${ }^{3}$ Department of Cardiology, The Hospital for Sick Children, Toronto, Canada.}

Published: 14 September 2011

doi:10.1186/1546-0096-9-S1-O14

Cite this article as: Venkatesan et al:: Clinical phenotype of neonatal lupus erythematosus relates to autoantibody level and gender. Pediatric Rheumatology 2011 9(Suppl 1):014.

\footnotetext{
* Correspondence: sharmila_venkatesan@hotmail.com

'Department of Rheumatology, Sophia's Children Hospital, Erasmus

University MC Rotterdam, The Netherlands

Full list of author information is available at the end of the article
} 